\title{
BEMERKUNGEN ZU EINEM MITHRAISCHEN RELIEF AUS TIBISCUM (JUPA, CARANSEBEŞ, RO) ${ }^{1}$
}

\author{
CĂLIN TIMOC*-IMOLA BODA** \\ * Banater Nationalmuseum zu Temeschwar, Rumänien \\ 300002 Timişoara, Huniade Str. 1 \\ calintimoc@gmail.com \\ ** Zentrum für römische Studien \\ „Babeş-Bolyai“-Universität zu Klausenburg, Rumänien \\ 400088 Cluj-Napoca, Napoca Str. 11 \\ boda_imola@yahoo.com
}

\begin{abstract}
Note on a Mithraic Relief from Tibiscum (Jupa, Caransebes, RO). Between pieces made of stone that in National Museum of Banat collection are preserved it is a Mithraic plate there, a fragment which has been cataloged in the literature with the wrong discovery place. This article aims to resume all discussions on this piece and to reconsider in a clearer context. So, after the notes from inventory-book, this piece comes through with certainty from the archaeological site Tibiscum (Jupa, Caransebeş, RO) and was a donation of a peasant named Ion Bogdea (villager from Jupa), offered to Marius Moga, the archaeologist who reopened at the Roman camp Jupa systematic archaeological research in 1964. After the analogies to this piece of Mithraic relief type we can say that it dates back somewhere in the late 2nd, beginning of 3rd century and was made in Colonia Sarmizegetusa, in the artistic stone workshop.
\end{abstract}

Keywords: miracle water scene, Mithraic relief, donation, Tibiscum

Unter den Steindenkmälern, die sich in der Sammlung des Banater Nationalmuseums zu Temeschwar (Timişoara) befinden, ist das Fragment eines mithraischen Reliefs bekannt, das nach der Meinung von G. Sicoe von einer siebenbürgischen archäologischen Fundstelle stammt, ${ }^{2}$ darauf weisen aber keine technischen Merkmale hin. Anlässlich der neuen, im Rahmen eines Digitalisierungsprojekts ${ }^{3}$ des operativen Archivs des Museums durchgeführten Überprüfung des Altbestandes wurde das Relief, das der Gegenstand der vorliegenden Untersuchung ist, wiederentdeckt und wir konnten es in einem neuen Kontext analysieren.

Gemäß des Registers des Banater Nationalmuseums zu Temeschwar (Abb. 1) stammt das Fundstück vom archäologischen Fundort Tibiscum (Jupa, Caransebeş, RO) und wurde von Ion Bogdea (vermutlich ein Bewohner aus Jupa) im Jahre 1964 in Vergesellschaftung von zwei römischen Ziegelsteinen Herrn M. Moga ${ }^{4}$ anlässlich des Neubeginns der systematischen archäologischen Forschungen geschenkt. ${ }^{5}$

Unter Beachtung der Tatsachen, dass die bisherigen Informationen weder beschreibend-technisch noch hinsichtlich der Bewertung des Reliefs innerhalb des mithraischen Kultes in Tibiscum richtig sind, haben wir es für notwendig gehalten, eine eingehende Analyse des Reliefs durchzuführen. Es ist nämlich wichtig, im Fall eines

\footnotetext{
${ }^{1}$ Diese Arbeit ist Teil des virtuellen Ausstellungsprojekts „Lapidarium 3D“ des Banater Nationalmuseums zu Temeschwar und wurde durch den lokalen Rat unterstützt. Die Arbeit wurde durch das Rumänische Nationale Amt für Wissenschaftliche Forschung, CNCSUEFISCDI, Projekt Nr. PNII-RU-TE-2014-4-0488 unterstützt.

${ }^{2}$ Als Erster führte G. Sicoe dieses Fundstück in den wissenschaftlichen Kreislauf ein. Weitere Informationen bei SICOE 2014, Nr. 220, Abb. 141
}

\footnotetext{
${ }^{3}$ Für mehrere Anmerkungen siehe: http://lapidarium3d. webs.com (letzte Aktualisierung am 11. 05. 2016).

${ }^{4}$ Marius Moga war Leiter des Banater Museums zu Temeschwar in den Jahren 1950-1974 und führte in Tibiscum (Jupa) die archäologischen Ausgrabungen zwischen den Jahren 1963 und 1975. Ausführliche Angaben findet man bei MedeLET 1993, 5-8.

${ }^{5}$ TUDOR 1968, 37.
} 


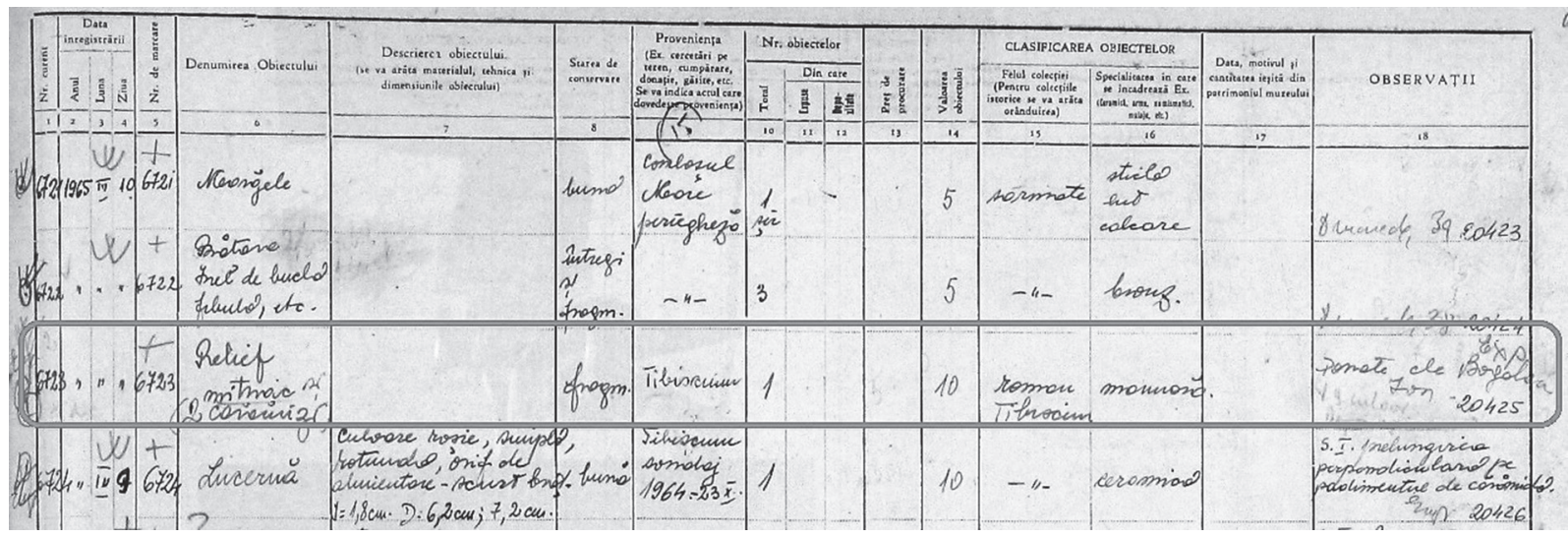

Abb. 1. Inventarregister des Banater Nationalmuseums zu Timișoara

Projekts alle Beweise dieses Kults für die Provinz Dakien (Supplementum CIMRM) zusammenzufassen und die Einordnung des vorliegenden Reliefs klar und richtig vorzunehmen. ${ }^{6}$

\section{DIE TECHNISCHEN DATEN DES RELIEFS (Abb. 2; $A b b .3)$}

1. Bezeichnung: mithraisches Relief, fragmentarisch erhalten.

2. Material, Technologie: graubläulicher Marmor (aus Bucova), als Flachrelief gemeißelt.

3. Konservierungszustand: Bruchstück; Stück des oberen Teils des Reliefs erhalten.

4. Maßangaben: Länge: 11,5 cm; Breite: 15,3 cm; Dicke: 2,7 cm; Breite des Randes und der Markierung, die das Feld teilt: $1,5 \mathrm{~cm}$.

5. Beschreibung des Fundstücks: Die Szene, die im oberen Feld erhalten blieb, nimmt $10 \mathrm{~cm}$ ein und aus der Szene der Taurochtonie blieb 3,2 cm erhalten.

6. Ikonographie: Der erhaltene Teil des Reliefs stellt eine Szene des Wasserwunders dar, in der Mithras mit einer phrygischen Mütze und einem wehenden Umhang auf dem Rücken in kniender Position (nur auf dem rechten Bein) erscheint, vor dem Felsen, der sich auf der rechten Seite des Flachreliefs befindet. Die Gestalt hält die rechte Hand in Richtung der Quelle und daran kann man den fließenden Wasserstrahl erkennen. (Aller Wahrscheinlichkeit nach haben wir mit der Szene des Wasserwunders zu tun.) Darüber, im Feld zwischen dem Kopf der Gestalt und der Ecke ihres Umhangs, kann man nach der Meinung von G. Sicoe einen Stier in einem Boot merken. ${ }^{7}$ Was man ganz klar erkennen kann, ist aber ein pflanzenfressendes Tier, mit unter sich geschlagenen Beinen im unteren Teil einer halbkreisförmigen Markierung. Im zentralen Relief wurde wahrscheinlich die Szene der Taurochtonie dargestellt.

7. Fundort: Tibiscum (Dacia Superior), Jupa, Caransebeş, Rumänien.

8. Aufbewahrungsort: Banater Nationalmuseum zu Temeschwar, MNB Inv.-Nr. 6723.

9. Zeitliche Einordnung des Fundstücks: Ende des 2. Jahrhunderts, Anfang des 3. Jahrhunderts n. Chr.

\section{ANALOGIEN}

Aus dem römischen Dakien stammt eine Reihe von Reliefs mit der Darstellung der Szene des Wasserwunders, die von G. Sicoe gesammelt und ausgelegt wurden. Bei dieser Gelegenheit möchten wir das behandelte Thema nicht wieder aufrollen, sondern wir versuchen möglichst viele Informationen über das zur Analyse vorgelegte Fundstück mit Hilfe einiger Analogien herauszufinden. Die Siedlungen, in denen solche Statuen zum Vorschein

${ }^{6}$ Wir sind unserem Kollegen Csaba Szabó (Universität zu

${ }^{7}$ SiCOE 2014, Nr. 220

Pécs und Erfurt) für die bibliographischen Anweisungen und die freundlichen Ratschläge sehr dankbar. 


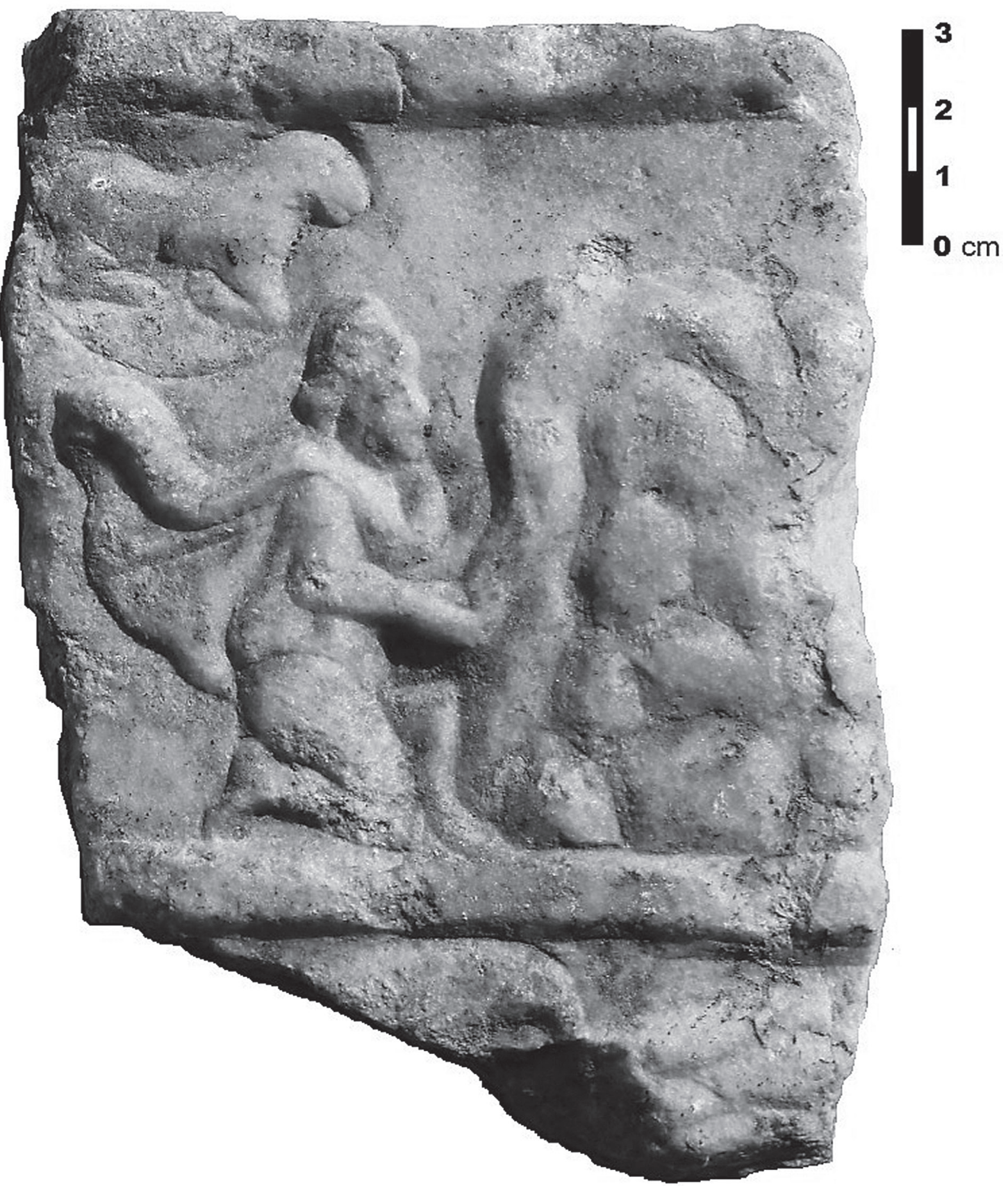

Abb. 2. Foto des mithraischen Reliefs von Tibiscum (nach C. Timoc), MNB Inv.-Nr. 6723 


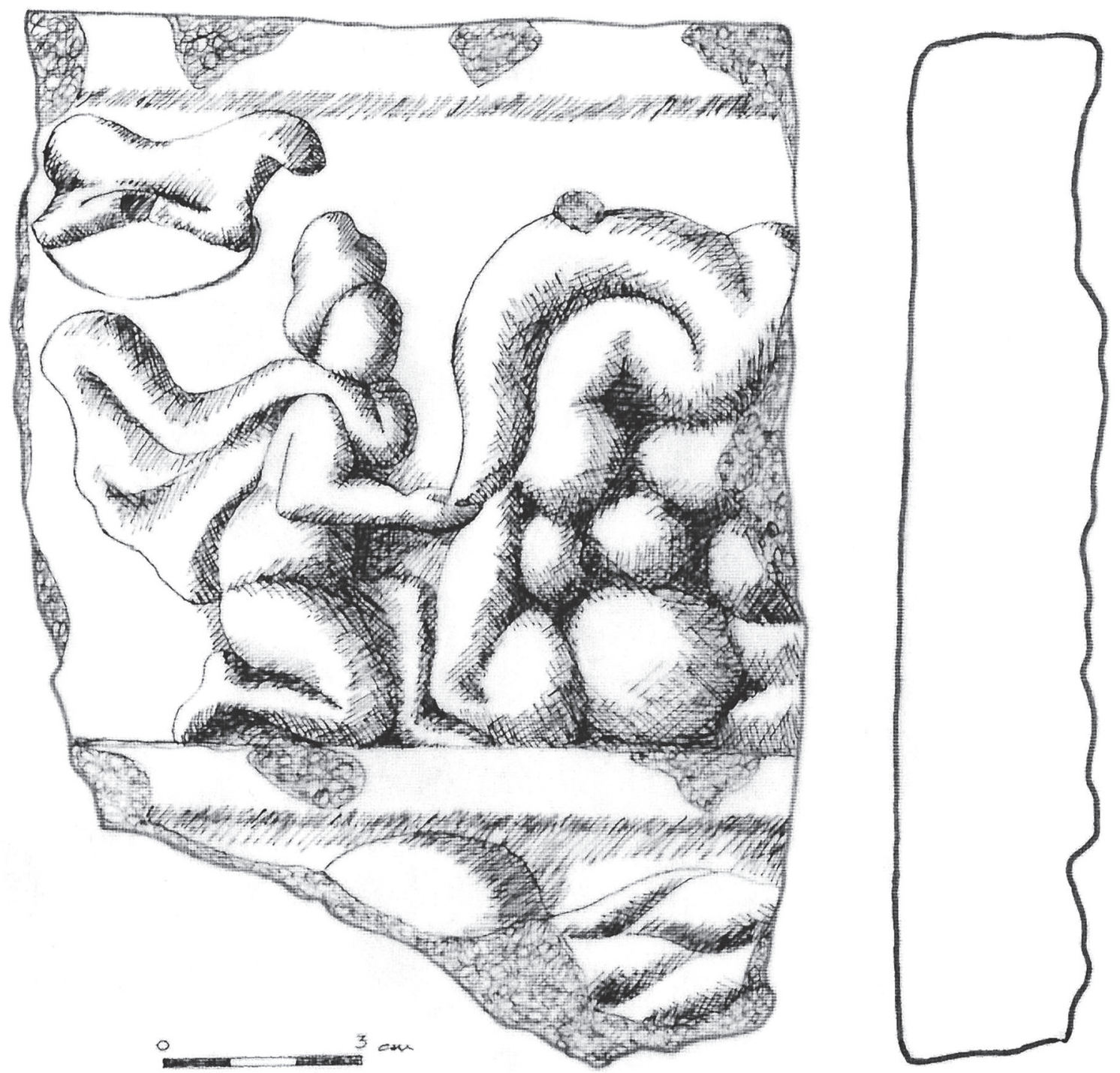

Abb. 3. Zeichnung des mithraischen Reliefs von Tibiscum (nach C. Timoc)

kamen, sind Colonia Sarmizegetusa/Sarmizegetusa (8 Fundstücke), ${ }^{8}$ Apulum/Alba Iulia (2 Fundstücke) ${ }^{9}$ bzw. je ein Fundstïck in Pojejena, ${ }^{10}$ Drobeta, ${ }^{11}$ Slăveni, ${ }^{12}$ Potaissa/Turda ${ }^{13}$ und Vințu de Jos. ${ }^{14}$

Aufgrund der 15 Beispiele (außer denen, die von uns präsentiert wurden) stellen wir die Tatsache fest, dass die erwähnte Szene immer im oberen Feld erscheint und die Anzahl der Wasserwunderdarstellungen zwischen den Feldern 2 und 3 variiert.

Mit Ausnahme von drei Fundstücken (Potaissa, ${ }^{15}$ Apulum, ${ }^{16}$ Vintu de Jos ${ }^{17}$ ) wurden alle anderen im Inneren der mithraischen Heiligtümer gefunden. Was die zeitliche Einordnung betrifft, konnte man sie nicht genau bestimmen. Man weiß nur, dass sie chronologisch zwischen der Mitte des 2. Jahrhunderts und dem 3. Jahrhundert einzuordnen sind.

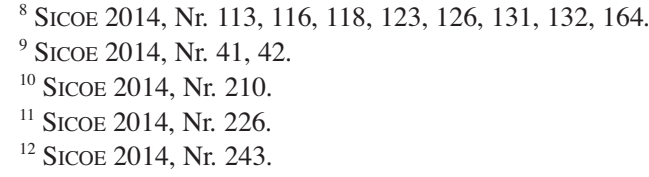

${ }^{8}$ Sicoe 2014, Nr. 113, 116, 118, 123, 126, 131, 132, 164

${ }^{9}$ SiCOE 2014, Nr. 41, 42

${ }^{10}$ Sicoe 2014, Nr. 210

${ }^{11}$ SiCOE 2014, Nr. 226.

${ }^{12}$ Sicoe 2014, Nr. 243.

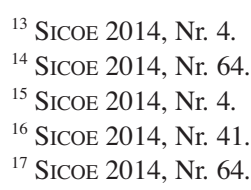


Nach der Darstellung der in der trajanischen Provinz ${ }^{18}$ bekannten Analogien möchten wir den Mithraskult in Tibiscum analysieren. Außer dem vorgestellten Relief steht ein epigraphisches Dokument zur Verfügung, das von Hermadio dem Gott Mithras für die Gesundheit des Publius Marius, der gemäß der Inschrift der Verwalter von Turranius Dius war, gewidmet wurde (Abb. 4). ${ }^{19}$

Erstens möchten wir den komplexen Namen der Gottheit hervorheben, da sein Ansprechen - wie es ersichtlich ist - nicht das gewöhnliche Ansprechen ist (Mithras, Sol Invictus Mithras), sondern führt er hier den persischen Namen Nabarzes. Dies ist kein ungewöhnliches Phänomen: Als Analogie erwähnen wir die Statuen aus Rom, ${ }^{20}$ Lucey in Gallia Narbonensis, ${ }^{21}$ Aquincum ${ }^{22}$ und eine andere von einem unbekannten Ort. ${ }^{23}$ All diese epigraphischen Dokumente beweisen, dass sich die Statue von Tibiscum unter die wenigen Beispiele einreihen lässt, bei denen der Gott sowohl lateinisch (invictus) als auch persisch (nabarzes) angesprochen wird. Gleichzeitig wird er mit dem Sonnengott der Römer (Sol, Helios) assimiliert, ${ }^{24}$ obwohl man nach den letzten Meinungen mit einem „Persianisierungsprozess“ des Namens des Gottes ${ }^{25}$ zu tun hat.

Zweitens möchten wir uns auf den Errichter der Statue, namens auf Hermadio, beziehen, der wahrscheinlich - jedenfalls seinem Namen nach - von griechischer Herkunft war. Diese Persönlichkeit ist in Dakien auch in Colonia Sarmizegetusa bekannt, wo er dem gleichen Gott einen Altar widmete. ${ }^{26}$ Es scheint, dass der Sklave in der mithraischen Hierarchie in Sarmizegetusa und Tibiscum eine große Rolle gespielt hatte; auf den epigraphischen Dokumenten nennt er Mithras Invictus, Anicetus und Nabarze. I. Tóth setzt voraus, dass wir Hermadio auch in Poetovio $^{27}$ und Rom $^{28}$ wiederfinden: Hier widmete er eine Statuengruppe, auf deren Inschrift wir das persische Ansprechen Nama finden. ${ }^{29}$

Ebenfalls in Tibiscum ist das sehr kleine Fragment einer Tafel bekannt, von der vorausgesetzt wird, dass sie mithraisch sei (Abb. 5). ${ }^{30}$ Leider kann man aus den erhaltenen Details weder einen ikonographischen Standpunkt bekommen noch aus dem erhalten gebliebenen Text darauf schließen, um welche Gottheit es sich handele. ${ }^{31}$

\section{SCHLUSSFOLGERUNGEN}

Mit Hilfe der Analyse des vorliegenden Reliefs und aller anderen Votivstatuen von Tibiscum sowie der analogen Fundstücke aus dem römischen Dakien können wir die Aufmerksamkeit auf verschiedene Aspekte lenken, die den bisherigen Standpunkt über das religiöse Leben in Tibiscum bestimmt ändern, ${ }^{32}$ und auf eine beschreibendtechnisch klarere Situation bezüglich des mithraischen Reliefs hinweisen können.

Erstens möchten wir hervorheben, dass die meisten Analogien mit der Darstellung der Wasserwunderszene aus der Colonia Sarmizegetusa stammen. Bis jetzt haben wir acht Fundstücke von hier. Daraus kann man eine einzige Schlussfolgerung ziehen: Außerhalb der Stadtmauern entstand die erste bzw. größte mithraische Gemeinschaft des römischen Dakiens. ${ }^{33}$ Gleichzeitig weiß man, dass es hier eine lokale Werkstatt gab, ${ }^{34}$ in der so gut wie einzig-

${ }^{18}$ BoDA-SZABÓ 2014, 110-115.

${ }^{19}$ IDR III/1, 145. Wie bei J.-R. Carbó García (CARBÓ GARCíA 2010, Nr. 67), lesen wir $n$ (umini?) anstatt $N($ abarze $)$ - eine Leseform, die von F. Cumont (Cumont 1893, 137, Nr. 293), M. Vermaseren (CIMRM 1956-1960, 323-324, Nr. 2153), M. Pintilie (PINTILIE 2003, 191, Nr. 74) und G. Sicoe (SiCOE 2014, Kat.-Nr. 203) nicht akzeptiert wurde. Der Altar ist im Banater Museum zu Temeschwar zu finden: MNB Inv.-Nr. 6357.

${ }^{20}$ CIL VI 742.

${ }^{21}$ CIL XII $2441=$ CIMRM I 915.

${ }^{22}$ CIL III 3481 = TitAq I, 256.

${ }^{23}$ CIMRM II 2197.

${ }^{24}$ NEMETI 2012, 149.

${ }^{25}$ GoRdON 2007, 392-405; GoRdON 2016.

${ }^{26} \mathrm{IDR}$ III/2, 283. Bemerkenswert ist die Wiederholung des Beinamens der Sieger: Es erscheint zunächst lateinisch als Invictus und zweitens

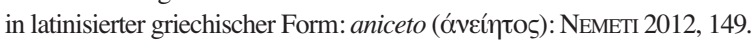

${ }^{27}$ CIMRM II 1582-3.

${ }^{28}$ CIMRM I 591.

${ }^{29}$ То́тн 1994, 153-160= То́тн 1995, 175-180= То́тн 2003, 81-86; NEMETI 2012, 149; SZABÓ 2015a.

${ }^{30}$ IsAC-STRATAN 1973, 121.

${ }^{31}$ IDR III/1, 147.

${ }^{32}$ BoDA-TiMOC 2016, im Druck.

${ }^{33}$ Die Gebäudepläne mit archäologischen Funden wurden von P. Király im Jahr 1886 veröffentlicht, in Zusammenhang mit der Beschreibung des Mithraeums von Sarmizegetusa (KIRÁLY 1886). Es wurde später von I. Boda (BoDA 2014, 307-351) in der Bewertung des 19. Jahrhunderts bezüglich des genannten Gebietes wieder publiziert. Die im Inneren des Heiligtums gefundenen kleinen Funde wurden schließlich von Cs. Szabó (SzABó 2014, 135-148) und die mithraischen Denkmäler von G. Sicoe analysiert (SiCOE 2014, 174-230, Nr. 72-194).

${ }^{34}$ SiCOE 2004, 285-302. 


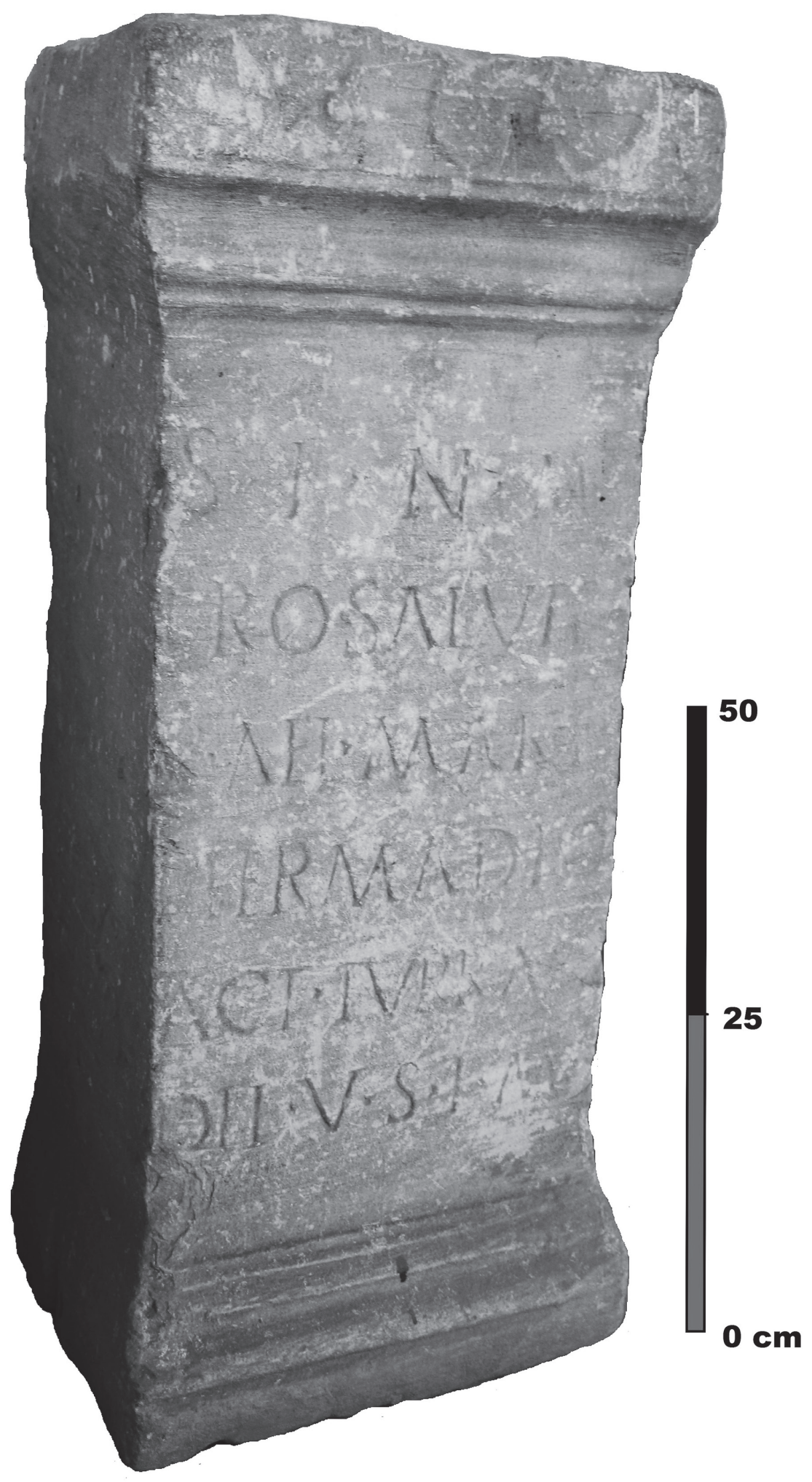

Abb. 4. Foto des Altars von Hermadio (nach C. Toma), MNB Inv.-Nr. 6357 

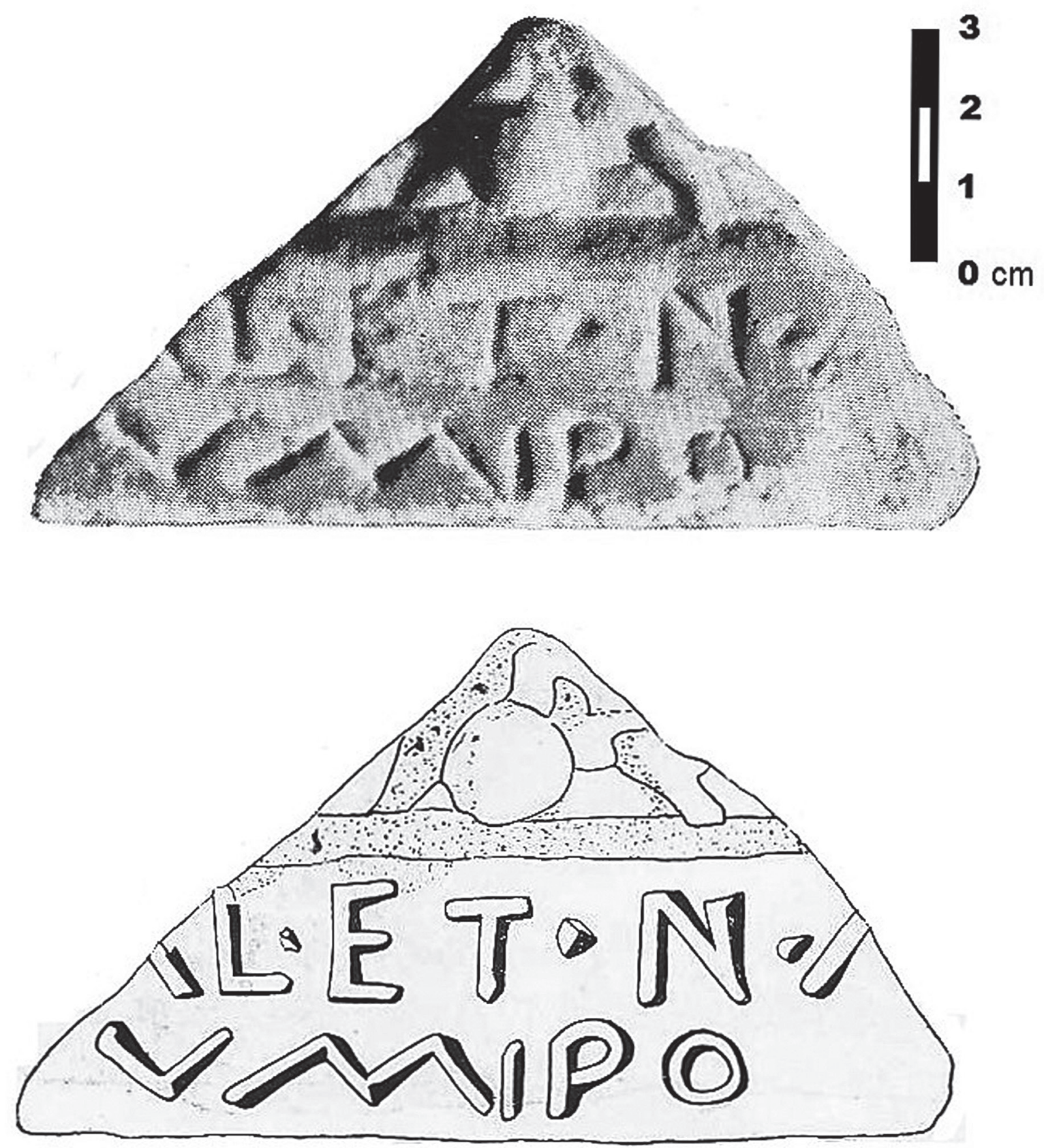

Abb. 5. Foto und Zeichnung des Reliefbruchstücks von Lugoj (nach IsAC-STRATAN 1973, Abb. 5) 
artige Stücke im römischen Reich hergestellt wurden. Dann verbreitete man sie in der trajanischen Provinz, vor allem in Apulum, das bis zur Regentschaft von Marcus Aurelius zum Territorium von Sarmizegetusa gehörte. ${ }^{35}$ Es ist wahrscheinlich, dass die erste mithraische Gemeinschaft in Apulum gegründet wurde, als Apulum zur colonia Sarmizegetusa gehörte. ${ }^{36}$ Diese Tatsache kann auch in Hinsicht von Tibiscum vorausgesetzt werden: Es ist nämlich bekannt, dass auch Tibiscum bis zum Ende des 2. Jahrhunderts zu Sarmizegetusa gehörte. ${ }^{37}$

Dazu wird die ,apostolische“ Tätigkeit von Hermadio in der zweiten Hälfte des 2. Jahrhunderts hinzugefügt. Wir wissen, dass er zuerst in der Colonia Sarmizegetusa und dann in Tibiscum tätig war. Das war die Periode der Gründung und Verbreitung des westlichen Mithraskults; diese Aufgabe leisteten insbesondere die Gründungspropheten und die missionierenden Apostel. ${ }^{38}$

Gemäß der überlieferten Analogien bezüglich des Hintergrundes der Entdeckung dieser Fundstücke kann gesagt werden, dass es auch in Tibiscum ein Heiligtum gegeben hat und es dem Gott Mithras gewidmet wurde. In diesem Sinne müssen der von Hermadio errichtete Votivaltar und auch seine ,,apostolische“ Tätigkeit erwähnt werden.

Schlussfolgernd unterstreichen wir die Tatsache, dass das vorliegende Relief, das ursprünglich aus zwei oder drei Feldern bestand, mit großer Wahrscheinlichkeit neben anderen ähnlichen Stücken in der Werkstatt der Colonia Sarmizegetusa hergestellt wurde. Die Errichtung des Heiligtums von Tibiscum und dadurch die Gründung der ersten mithraischen Gemeinschaft gehen in die Zeit der Tätigkeit von Hermadio zurück. Das ist zugleich die Zeit, als die Siedlung Tibiscum Teil des Gebietes von Sarmizegetusa war.

\section{LITERATUR}

ARDEVAN 1998

BESKOW 1980

BODA 2014

BODA-SZABÓ 2014

BoDA-Timoc 2016

Carbó García 2010

Cumont 1893

DoвÓ 1940

GORDON 2007

GORDON 2016

ISAC-STRATAN 1973

KIRÁLY 1886

Medeleț 1993

Nemeti 2012

Pintilie 2003

PISO 1995
= R. ARDEVAN: Viata municipală în Dacia romană. Timișoara 1998.

= P. BesKow: The Portorium and the mysteries of Mithras. Journal of Mithraic Studies III.1-2 (1980) $1-18$.

= I. BoDA: Ulpia Traiana Sarmizegetusa and the archaeological research carried out between 1881 and 1893. Studia Antiqua et Archaeologica 20 (2014) 307-351.

= I. BoDA-Cs. SzABÓ: The Bibliography of Roman Religion in Dacia. Cluj-Napoca 2014.

= I. BoDA-C. TIмос: The Sacred Topography of Tibiscum. Studia Universitatis Babeș-Bolyai - Historia 60/1 (2016). Im Druck.

= J.-R. CARBó GARCÍA: Los cultos orientales en la Dacia Romana. Formas de difuzión, integración, y control social e ideológico. Salamanca 2010.

= F. Cumont: Texts et Statues figurés relatifs aux Mystères de Mithra II. Paris 1893.

= Á. DoBó: Publicum Portorium Illyrici. Budapest 1940.

= R. Gordon: Institutionalized Religious Options: Mithraism. In: J. Rüpke (ed.): A Companion to Roman Religion. Oxford 2007, 392-405.

$=$ R. Gordon: Persie in spelaeus Solemn colunt: Mithra(s) between Persia and Rome. In: R. Strootman-M. J. Versluys (eds): Persians in Antiquity. Stuttgart 2016.

= D. IsAC-I. STRATAN: Monumente de artă provincială romană în Muzeul din Lugoj. Banatica 2 (1973) 120-123.

= P. KIRÁLY: A sarmizegetusai mithraeum [Das Mithraeum von Sarmizegetusa]. Budapest 1886.

= FL. Medeleț: Profesorul Marius Moga la cea de-a 80-a aniversare. Analele Banatului S.N. 2 (1993) $5-8$.

= S. NEMETI: Dialoguri păgâne. Formule votive şi limbaj figurat în Dacia romană. Iaşi 2012.

= M. Pintilie: Mithrae în Dacia. Ph.D. Thesis. Cluj-Napoca 2003.

= I. PISO: Le territoire de la Colonia Sarmizegetusa. Ephemeris Napocensis 5 (1995) 63-82.
${ }^{35}$ Piso 2005, 273-295; SicOe 2014, 126; SZABÓ 2015b, 6.

${ }^{36}$ SZABÓ 2015b, 6.

${ }^{37}$ Piso 1995, 63-82; Ardevan 1998, 74.

${ }^{38}$ Tóтн 2003, 81-86. Publicum Portorium Illyrici und die Angestellten (conductor, procurator, vilicus, vicarius vilici, contrascriptor, contrascriptor ex vicario, arcarius, vicarius arcari, scrutator, tabularius - mehrere Informationen siehe bei DoBó 1940, 157-161). Er hatte eine wichtige Rolle bei der Verbreitung des Mithraismus (BESKOw 1980, 1-18; Tо́тH 1977, 385-392; CARBó GARCíA 2010, 165). Eine direkte Verbindung zwischen Portorium und Dakien bezieht sich auf einige Personen wie Q. Sabinius Veranus, C. Antonius Rufus und T. Iulius Saturninus. Mehrere Informationen siehe bei Dobó 1940, 168-169; PISO-Moga 1998, 105-108; SzABó 2015a, 407-422. Diejenige, die als „Missionäre“ in der Verbreitung des Mithraskultes in Betracht gezogen werden können, sind T. Aurelius Marcus (CIMRM 1959, 1960), Aurelius Maximus, M. Aurelius Timotheus und Eutyches libertus (CIMRM 1935, 1937), Dioscorus des Marcus (Szabó-Boda-Bunoiu-Timoc 2016, im Druck), M. Valerius Maximianus wie auch C. Iulius Castinus (SZABó 2015a, 407-422). 
PISO 2005

PISO-Moga 1998

SiCOE 2004

SICOE 2014

SzABó 2014

SZABÓ 2015a

SZABÓ 2015b

SzABÓ-BodA-BunOIU-TIMOC 2016

TóTH 1977

То́тн 1994

TóTH 1995

TÓTH 2003

TUdOR 1968

VERMASEREN 1956-1960
= I. PISO: An der Nordgrenze des Römischen Reiches. Stuttgart 2005

= I. PISO-V. MogA: Un bureau du Publicum Portorium Illyrici à Apulum. ActaMN 35/1 (1998) 105-108.

= G. SicoE: Lokalproduktion und Importe. Der Fall der mithraischen Reliefs aus Dakien. In: M. Martens-G. De Boe (eds): Roman Mithraism: The Evidence of the Small Finds. Brussel 2004, 285-302.

= G. SICOE: Die mithräischen Steindenkmäler aus Dakien. Cluj-Napoca 2014.

= Cs. Szabó: Notes on the Mithraic small finds from Sarmizegetusa. Ziridava, Studia Archaeologica 28 (2014) 135-148.

= Cs. Szabó: The Cult of Mithras in Apulum: Individuals and communities. In: L. Zerbini (ed.): Atti di Convenio II. International Conference on Danubian Provinces, Ferrara, 20-22. November 2013 Ferrara 2015, 407-422.

= Cs. SzABó: Cautes bikafejjel: egy ritka ikonográfia nyomában [Cautes mit Stierkopf: auf der Spur einer seltenen Ikonographie]. Erfurt 2015.

= Cs. Szabó-I. Boda-V. Bunoiu-C. Timoc: Notes on a new Mithraic inscription from Dacia. In R. Ardevan, E. Beu-Dachin (eds): Mensa rotunda epigraphiae Daciae Pannoniaeque. Cluj-Napoca 2016. Im Druck.

= I. Tóтн: Das lokale System der mithraischen Personifikationen im Gebiet von Poetovio. Archeološki Vestnik 28 (1977) 385-392.

= I. То́тн: A Dacian "Apostle" of the cult of Mithras? Specimina Nova 8 (1994) 153-160.

= I. Tóтн: A Dacian “Apostle" of the cult of Mithras? Din istoria Europei romane, Seria Istoria 4. Oradea 1995, 175-180.

= I. Tóth: Mithras Pannonicus. Budapest-Pécs 2003.

= D. TudoR: Orașe, târguri si sate în Dacia romană. București 1968.

= M. VERMASEREN: Corpus Inscriptionum et Statueorum Religionis Mithraicae (CIMRM) I-II. Hague 1956-1960. 